\title{
ACROMEGALIA
}

\section{ASPECTOS DIAGNÓSTICOS E TERAPÊUTICOS}

\author{
Análise de 18 casos
}

\section{FLÁVIO FREINKEL RODRIGUES*, ALICE HELENA DUTRA VIOLANTE**,

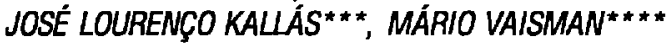

\begin{abstract}
RESUMO - Os autores fizeram estudo retrospectivo de 18 pacientes com acromegalia, que foram diagnosticados, tratados e acompanhados pelos Serviços de Endocrinologia e Neurocirurgia do Hospital Universitário Clementino Fraga Filho da Universidade Federal do Rio de Janeiro, no período de 1979 a 1985 . A média de idade dos pacientes foi 43,2 anos (variação entre 15 e 63), as queixas clínicas iniciais se deveram principalmente à alteraçōes somáticas $(83,33 \%)$, em $50 \%$ dos casos tivemos manifestações secundárias à compressão tumoral e ern 53,33\% havia alteraçōes neuro-oftalmológicas. A comprovação de hipersecreção de hormônio de crescimento foi feita por dosagens hormonais basais e por testes dinârnicos. A avaliação neuro-radiológica evidenciou expansão suprasselar em $61,11 \%$ dos casos e em $100 \%$ dos casos o acesso cirúrgico foi por via transesfenoidal. O objetivo principal deste estudo foi o de estabelecer critérios para o diagnóstico, discutir a conduta terapêutica e avaliar os resultados obtidos, comparando-os com outras séries da literatura.
\end{abstract}

PALAVRAS-CHAVE: acromegalia, diagnóstico, terapêutica.

\section{Acromegaly: diagnostic and therapeutic aspects. Analysis of 18 cases}

ABSTRACT - The authors present a retrospective study of a series of eighteen patients with acromegaly diagnosed. treated and followed by the Endocrinology and Neurosurgery Services of the Hospital Universitário Clementino Fraga Filho of the Federal University of Rio de Janeiro. The average age of the patients was $43.2 \%$ years (varying between 15 and 63). Initial complaints were mainly due to somatic alterations in $83.33 \%$; half the cases had manifestations secondary to tumor compression and $53.33 \%$ had neuro-ophtalmological alterations. Hypersecretion of growth hormone was demonstrated by basal hormone determinations and dynamic tests. Neuroradiological assessment showed supraselar expansion in $61.11 \%$ of cases. Surgical approach was transsphenoidal in all cases. The main objetive of this study was to establish diagnostic criteria, discuss the therapeutic conduct and evaluate the results obtained, comparing them with other series of literature.

KEY WORDS: acromegaly, diagnosis, therapy.

Acromegalia é doença causada pelo excesso de produção de hormônio de crescimento (HGH) em indivíduos após a puberdade. Quando esta hipersecreção ocorre na infância é chamada de

Estudo realizado nos Serviços de Endocrinologia e de Neurocirurgia do Hospital Universitário Clementino Fraga Filho (HUCFF) da Universidade Federal do Rio de Janeiro (UFRJ): *Chefe do Serviço de Neurocirurgia do HUCFF, Professor Adjunto de Neurocirurgia da Faculdade de Medicina UFRJ; **Professora Adjunta de Endocrinologia da Faculdade de Medicina da UFRJ; ***Médico do Serviço de Neurocirurgia do HUCFF da UFRJ; **** Chefe do Serviço de Endocrinologia da HUCFF, Professor Adjunto de Endocrinologia da Faculdade de Medicina da UFRJ. Aceite: 10-outubro-1996.

Dr. Flávio Freinkel Rodrigues - Rua Assunçāo 162 apto. 501 - 22251-030 Rio de Janeiro RJ - Brasil. 
gigantismo. Geralmente sua etiologia é um tumor hipofisário que por imuno-histoquímica evidencia células produtoras de HGH. Pode ocorrer de forma isolada ou, como em tumores, em que se associa a presença de células produtoras de prolactina ${ }^{6}$. A quantidade de grânulos secretores de HGH no tumor, detectada por microscopia eletrônica, não está relacionada aos níveis plasmáticos de HGH, mas sim com o grau de diferenciação e crescimento tumoral. Algumas pessoas apresentam manifestações clínicas de acromegalia sem quadro laboratorial e ausência de comprovação neuroradiológica, justificando a denominação de acromegaloidismo, por Mins, citado por Michael e col. ${ }^{11}$. A morbidade da doença origina-se dos efeitos periféricos causados pela quantidade aumentada do HGH ou do efeito de massa local, com compressāo de estruturas vizinhas pelo tumor. Dependendo do estágio da doença e do tamanho, forma e grau de agressividade do tumor, os efeitos periféricos ou as manifestações locais compressivas podem dominar o quadro clínico.

A acromegalia é afecção crônica e debilitante, caracterizada por alterações cosméticas e anormalidades metabólicas, e, frequentemente, cursa com alterações de vários órgãos (artropatia, cardiomiopatia e neuropatia) ${ }^{2}$. As doenças cardiorrespiratórias constituem a principal causa da morte. Estas taxas devido à doença cardiovascular, são 5 a 10 vezes mais elevadas que na população geral ${ }^{2}$. Também parece haver aumento da incidência de doença maligna em pacientes acromegálicos ${ }^{7}$. Portanto, é da maior importância estabelecer o diagnóstico e tratar o paciente acromegálico em franca atividade de doença, o mais precocemente possivel. As alternativas de terapia são: cirurgia, irradiação e tratamento clínico. Sabe-se que, apesar dos bons resultados da farmacoterapia em vários pacientes ${ }^{7}$, há limitações quanto ao tratamento clínico, ou pela resposta insatisfatória a certas drogas (agonistas dopaminérgicos) ou pelos efeitos colaterais que estas substâncias apresentam (agonistas da somatostatina) ou ainda pelos altos custos das medicações em nosso meio, o que muitas vezes inviabiliza esta opçāo. A cirurgia se impōe como terapêutica de escolha, por ser rápida e eficaz e, em muitos casos, dispensando terapia complementar. A radioterapia é considerada principalmente como tratamento coadjuvante, sendo utilizada como principal opção em situaçōes especiais. como por exemplo em pacientes com alto risco cirúrgico.

Ao avaliarmos este grupo de pacientes acromegálicos, procuramos enfatizar os aspectos de diagnóstico, tanto clínico como complementar, as alternativas terapêuticas e o prognóstico naqueles que se mantiveram sob os nossos cuidados.

\section{MATERIAL E MÉTODOS}

Estudamos, retrospectivamente, 18 pacientes acromegálicos (14 mulheres e 4 homens), que foram submetidos a tratamento neurocirúrgico, após avaliação pré-operatória pelo Serviço de Endocrinologia do HUCFF da UFRJ, no período de 1979 a 1985.

A comprovação endocrinológica de atividade acromegálica foi feita através de:

- níveis basais de HGH: níveis médios menores que $2 \mathrm{ng} / \mathrm{dl}$;

- TOTG/HGH (teste oral de tolerância a glicose corn dosagem de hormônio de crescimento): é considerada resposta normal ao teste a queda de HGH para níveis menores que $2 \mathrm{ng} / \mathrm{dl}$ após a sobrecarga glicídica; nos acromegálicos pode ocorrer não só a falta de supressão, como resposta paradoxal com aumento dos níveis de $\mathrm{HGH}$;

- teste do TRH/HG (teste de estímulo de fator hipotalâmico estimulante da secreção de células tireotroficas, TRH, com dosagem de hormônio de crescimento): no indivíduo normal não há aumento dos níveis de HGH, o que ocorre na acromegalia, evidenciando resposta anômala.

Todas as dosagens hormonais foram feitas por radioimunoensaio (RIA), CIS, com valores nornais até $14 \mathrm{ng} / \mathrm{dl}$.

O diagnóstico por imagens foi feito através de $\mathrm{RX}$ de crânio, tomografia helicoidal além de tomografia computadorizada de crânio (TC), e em alguns foi possível ser realizada ressonância magnética de crânio (RNM). 


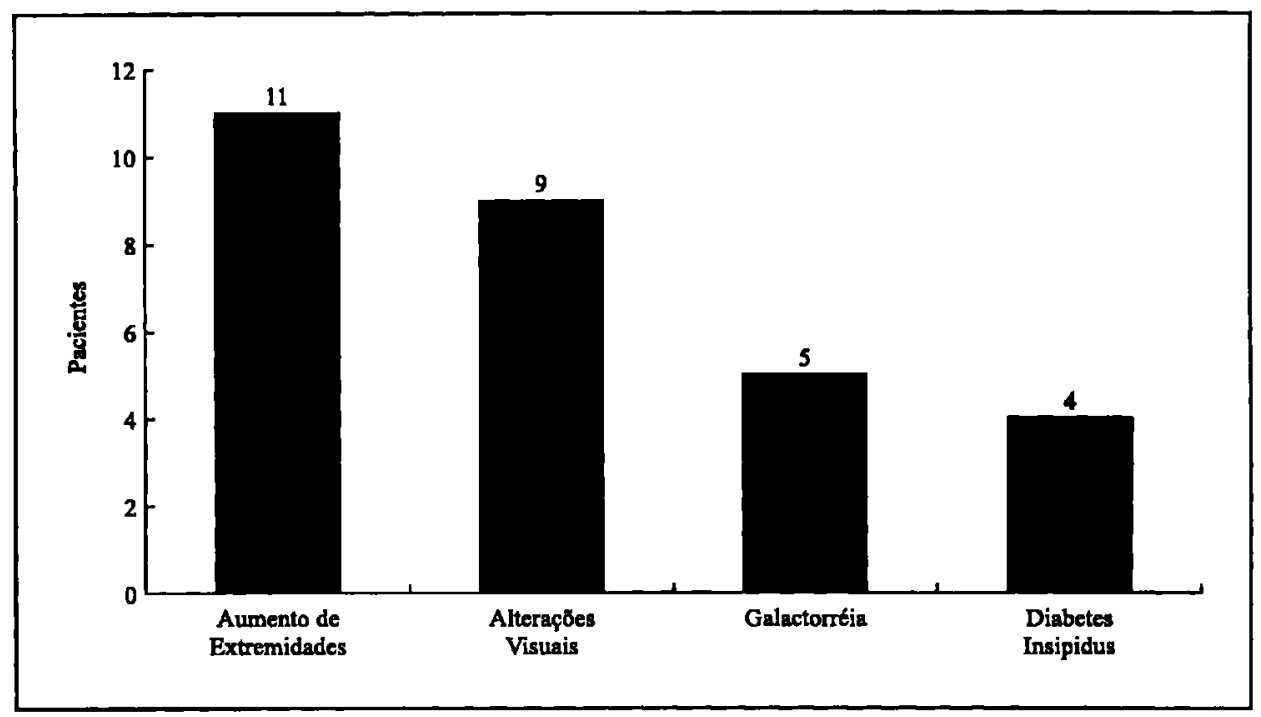

Fig 1. Acromegalia: aspectos clínicos (principais sinais e sintomas).

\section{RESULTADOS}

A média de idade de nossos pacientes foi 43,2 anos (variação de 15 para 59) para as mulheres e de 49,5 anos (variação de 30 a 63 anos) para os homens.

O motivo da consulta em $83,33 \%$ dos casos foi devido às alteraçōes somáticas de hipersecreção de HGH, estando as queixas relativas ao efeito compressivo do tumor (cefaléia e/ou alterações visuais) presentes em nove pacientes, (50\%) dos indivíduos avaliados. Os pacientes foram submetidos a campimetria pré-operatória, com alteração da mesma em oito casos $(44,44 \%)$ e as restante normais (Fig 1).

$\mathrm{Na}$ investigação diagnóstica da atividade acromegálica, o nível médio de $\mathrm{HGH}$ plasmático foi $72,07 \mathrm{ng} / \mathrm{dl}$ (variando de 10,4 a 20,7) antes da cirurgia. No pós-operatório, este valor foi de 13,6 ng/ dl (variou de 0,20 a 73) com queda média em tomo de 5 vezes dos valores iniciais (Fig 2). Os testes de TRH/HGH e/ou TOTG/HGH apresentaram resposta anormal de HGH em 12 pacientes $(66,66 \%)$.

A análise neuro-radiológica evidenciou tumor hipofisário em todos os casos, com expansão suprasselar em $11(61,11 \%)$.

O acesso cirúrgico se deu por via trans-oro-naso-esfenoidal (transesfenoidal) nos 18 pacientes. As complicações relatadas foram: sinusite clínica em 3 casos $(17,64 \%)$, diabetes insipidus central em $2(11,76 \%)$ e hipogonadismo em $1(5,5 \%)$. Sete tiveram recidiva das manifestações de acromegalia $(41,17 \%)$ e todos se submeteram a tratamento complementar: radioterapia isolada em $7(38,88 \%)$, entre estes a paciente que nāo pode ser submetida a retirada do tumor; radioterapia e bromocriptina em 1 paciente com evidência de recidiva $(5,88 \%)$ e em outro em que houve retirada parcial do tumor (Fig 3 ).

\section{DISCUSSĀO}

No grupo de acromegálicos estudados, encontramos predomínio de alteraçōes da hipersecreção de HGH como motivo da consulta, e uma alta incidência, quando comparado a literatura, de alteraçōes visuais $\left(53,33 \%\right.$ de nossos casos e $20 \%$ como valor médio relatado por diversos autores ${ }^{1,7}$ ).

$\mathrm{Na}$ presente série, houve relato de hiperprolactinemia em $44,44 \%$ dos casos, 8 pacientes. $\mathrm{Na}$ glândula hipofisária normal, o HGH e a prolactina são produzidos por diferentes tipos celulares. A 


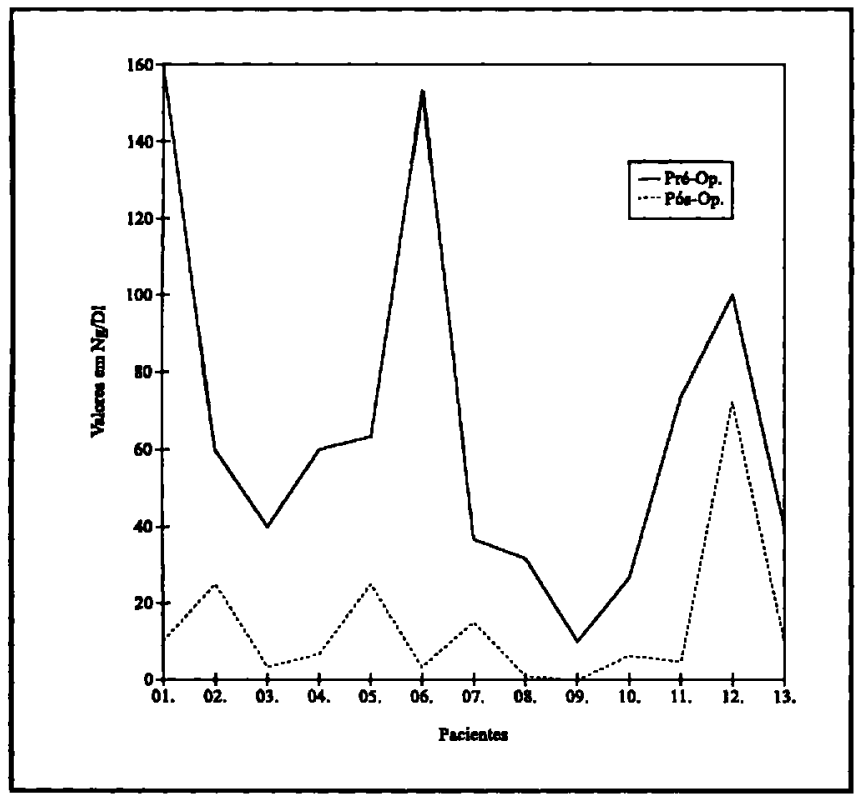

Fig 2. Comprovação da atividade laboratorial acromegálica (dosagem de GH no pré e pós-operatório).

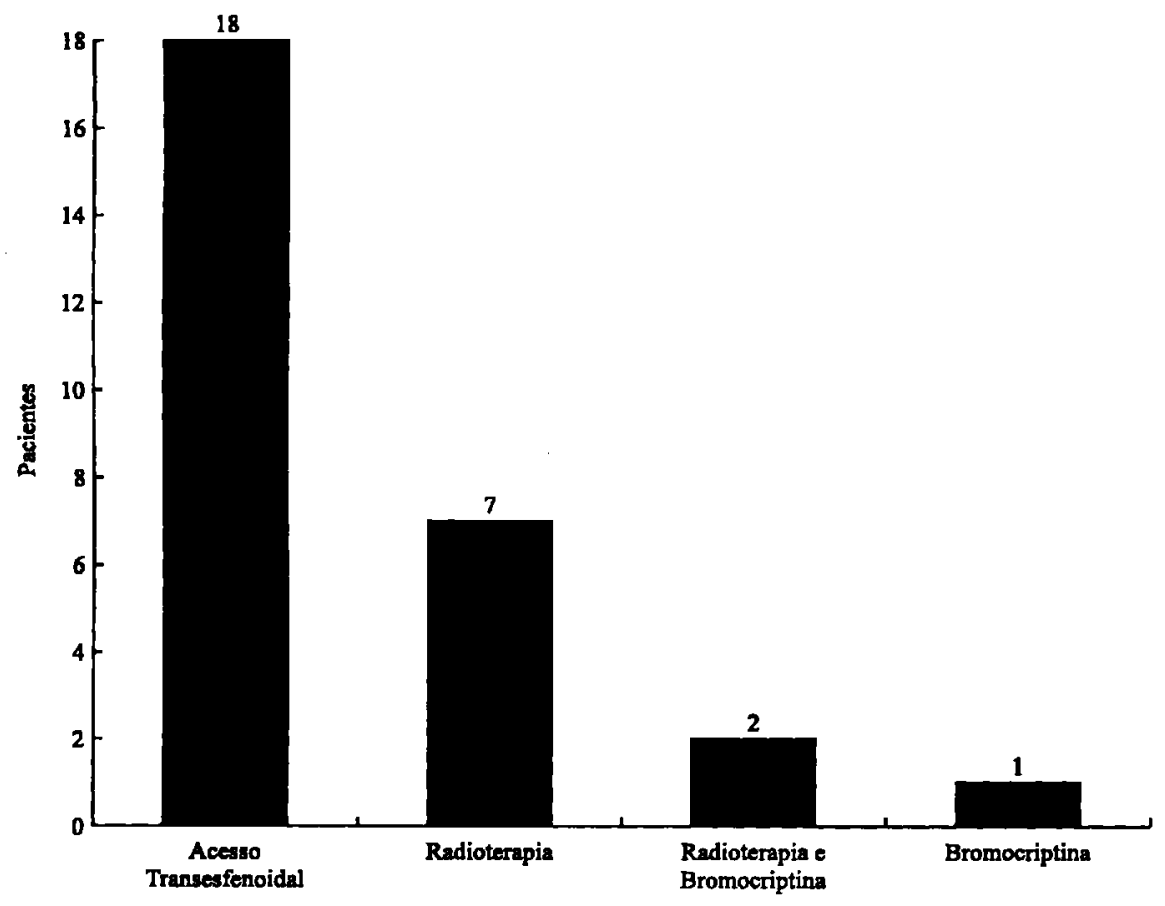

Fig 3. Acromegalia: conduta terapêutica e tratamento complementar. 
cultura de tecidos e os métodos de imuno-histoquímica têm demonstrado que os adenomas de muitos pacientes acromegálicos contêm tanto HGH como prolactina ${ }^{1,2}$. Em pacientes com este tipo de adenoma, alguns pesquisadores concluiram que estes hormônios podem ou não ser liberados por um mesmo tipo de célula ${ }^{2}$, havendo inclusive hiperprolactinemia em tumores produtores apenas de HGH, por causa até o momento inexplicada ${ }^{7}$. Para Kanie e col. ${ }^{9}$, o aumento da prolactina e do HGH estão presentes simultaneamente em 30 a $60 \%$ dos pacientes acromegálicos, para Daughaday $\mathrm{em}^{20}$ a $40 \%$ e cerca de $35 \%$ para Frohman?.

O diagnóstico de acromegalia $\mathcal{E}$ feito pela demonstraçāo de níveis plasmáticos de $\mathrm{HGH}$ aumentados e resposta anormal aos testes funcionais. Os níveis basais de HGH são muito variáveis, com valores desde normais até extremamente aumentados. O padrão secretório do $\mathrm{HGH}$ em acromegálicos é diferente do encontrado em pessoas normais ${ }^{3} \mathrm{e}$ isto auxilia na avaliação clínica pré e pós-operatória da funcionalidade tumoral (atividade acromegálica). Utilizamos em nossos pacientes o TOGT, que é bastante sensível, com resposta anormal do HGH em tomo de 70 a 80\% dos indivíduos das diversas séries relatadas? ${ }^{7}$. Usamos também a resposta do HGH ao TRH, que apresenta sensibilidade semelhante ao TOTG. Dos 18 pacientes submetidos a avaliação de atividade acromegálica, todos apresentaram um ou ambos os testes alterados e após a cirurgia houve normalização destes em 8 $(44,44 \%)$. Portanto, a constatação de atividade acromegálica, dado importante para mostrar que há naquele momento hipersecreção de HGH, foi evidenciada em nossos pacientes no pré e esteve ausente em um grupo no pós-operatório, pela dosagem de HGH e pelo TOTG/HGH e/ou pelo teste do TRH/ HGH. Naqueles em que havia evidência desta atividade, houve indicação de terapia complementar a cirurgia.

O procedimento diagnóstico por imagem de escolha para acromegalia é a RNM com o uso do constraste paramagnético (gadolíneo), que deve ser usado principalmente na suspeita de microadenomas. Apesar de não apresentar o grau de resolução da RNM, a TC com cortes coronais é bastante útil no diagnóstico e caracterização destes tumores. Foi o método de imagem predominantemente utilizado em nossos pacientes $(83,33 \%)$, e evidenciou expansão supraselar em $61,11 \%$ dos casos.

Atualmente é inquestionável que o tratamento de escolha para os tumores que produzem excesso de HGH é a cirurgia transesfenoidal. Esta, além de eliminar o efeito de massa do tumor, inibe a hiperatividade endócrina e melhora a função pituitária, alcançando estes objetivos imediatamente e com o mínimo de morbidade. Dos nossos pacientes, $44,44 \%$ não apresentaram recidiva da acromegalia e não foram submetidos a tratamento complementar, dados coincidentes com os de Barkan'.

A falta de resposta satisfatória ao tratamento cirúrgico e à radioterapia, principalmente naqueles pacientes com grandes adenomas hipofisários, enseja o uso de várias substâncias com o objetivo de inibir a secreção aumentada de HGH. Em nosso meio o uso mais frequente é da bromocriptina, derivado do ergot, com potente atividade como agonista dopaminérgico, assim como o seu similar pergolide, que agem diretamente sobre o tumor ${ }^{4.7 .15}$. A resposta a esta terapia é variável, se situando entre 25 a $50 \%$ dependente da concentraçāo de receptores para dopamina no tumor. Oppizzi e col. ${ }^{13}$ mostraram uma série de acromegálicos que usaram os agonistas da dopamina por longo prazo e obtiveram redução de $50 \%$ dos níveis de HGH plasmático. Estes e outros autores mostraram também redução do tamanho do tumor $, 7,7,10$.

A dose utilizada é alta, se situando entre 15 a $60 \mathrm{mg}$ ao dia, o que aumenta bastante o custo do tratamento. Embora o efeito colateral mais comum sejam náuseas que tendem a desaparecer com o uso contínuo, vários pacientes apresentaram intolerância severa a estes fármacos ${ }^{7}$. A retirada da medicação faz com que haja recidiva clínica e laboratorial. Apenas $3(16,66 \%)$ dos pacientes desta série utilizaram bromocriptina como terapia complementar a cirurgia e/ou radioterapia, o que $\epsilon$ justificado pelo baixo poder aquisitivo da maioria. 
Para alguns autores, como Oppizzi e col. ${ }^{13}$ e Spinas e col. ${ }^{15}$, o resultado da cirurgia transesfenoidal foram bons, mas o prognóstico foi pobre naqueles pacientes com altos níveis de HGH préoperatórios e nos tumores com extensão suprasselar. Remoção incompleta do tumor mais irradiação pós-operatória tem alta probabilidade $(95 \%)$ de prevenir a recidiva tumoral, mas o risco da irradiação induzir hipopituitarismo é muito elevado.

$\mathrm{Na}$ série que estudamos, 9 pacientes foram submetidos a radioterapia (50\%), por ressecção incompleta do tumor e nenhum dos pacientes desenvolveu hipopituitarismo nos primeiros 6 meses de avaliaçăo. Observou-se, também, nesta série, que apenas $3(16,6 \%)$, apresentaram como complicaçāo sinusite maxilar e $2(11,1 \%)$, diabetes insípidus central transitório. Acreditamos que a baixa taxa destas complicaçōes se deveu a ressecçāo subtotal em grande número dos nossos pacientes.

Apesar de sua rapidez e eficácia, o sucesso da cirurgia para acromegalia é bastante variável, dependendo do tamanho do tumor e do critério de "cura". Consideramos queda dos níveis de HGH basal para valores menores que $2 \mathrm{ng} / \mathrm{dl}$ e/ou resposta normal aos testes de TRH e TOTG ${ }^{7}$. As complicaçōes pós-operatórias tardias estāo dentro do relatado na literatura internacional ${ }^{6,7.14}$.

\section{CONCLUSÕES}

Apesar do número reduzido de pacientes, nossos resultados são comparáveis com os descritos na literatura internacional;

O tratamento cirúrgico é a terapia de eleiçăo para os pacientes com acromegalia;

A radioterapia e as drogas sāo terapêutica coadjuvante na maioria dos casos, só assumindo o papel principal no tratamento dos poucos casos em que a cirurgia é contra-indicada;

O diagnóstico precoce é muito importante, já que melhora o prognóstico e a qualidade de vida dos pacientes.

\section{REFERENCIAS}

1. Barkan AL. Acromegaly: diagnosis and therapy. Endocr Metab Clin North Am 1989;18:277-309.

2. Baumann G. Acromegaly and pituitary tumors: diagnosis and management. Endocr Metab Clin North Am 1987;16:685-703.

3. Berg GG, Frolich M, Veldhuis JD, Roelfesema F. Growth hormone secretion in recently operated acromegalic patients. J Clin Endocrinol Metab 1984;79:1705-1715.

4. Chanson PH, Timsit J, Harris AG. Octreotide analogue de la somatostatine (proprietés pharmacologiques et applications thérapeutiques dans les tumeurs endocrine-hypophysaires). Presse Méd 1993;40:2009-2013.

5. Daughaday WH. Growth-hormone insulin-like growth factor and acromegaly. In De Groot LJ (ed). Endrocrinology - Ed 3. Philadelphia: Saunders, 1995:303-329.

6. Fahlbusch R, Honneger J, Buchfelder M. Surgical management or acromegaly. Endrocr Metab Clin North Am 1992;21:669-679.

7. Frohman LA. Diseases of the anterior pituitary. In Felig P, Baxter JD, Frohman LA (eds). Endocrinology and Metabolism. Ed 3. New York: MacGraw-Hill, 1995:289-383.

8. Jackson LMD, Barnard LB, Lamberton P. Role of a long-acting somatostatin analogue (SMS 201-995) in the treatment of acromegaly. Am J Med 1986;81:94-101.

9. Kanie N, Kagena N, Kuwayama A. Pituitary adenoma in acromegalic patients: an immunohistochemical and endocrinological study with special reference to prolactin secreting adenoma. J Clin Endocrinol Metab 1983;52:272-276.

10. McKinght JA, McCance DR, Sheridan B, Atkinson AB. Four years treatment of resistant acromegaly with octreotide. Eur J Endocrinol 1995;132:429-432.

11. Michael W, Ashcraft T, Pamela I. A unique growth factor in patient with acromegaloidism. J Clin Endocrinol Metab $1983 ; 52: 272-276$.

12. Newton DR, Dillon WP, Norman D. Gadolinium DTPA-MR imaging of pituitary adenomas. AJNR no prelo.

13. Oppizzi G, Petroncini NM, Dallabonzana D. Relationship between somatomedin C and growth hornone levels in acromegaly: basal and dynamic evaluation. J Clin Endocrinol Metab 1986;63:1348-1353.

14. Ross DA, Wilson CB. Results of transsphenoidal microsurgery for growth hormone secreting pituitary adenoma in a series of 214 patients. J Neurosurg 1988;68:854-866.

15. Spinas GA, Sapf J, Landolt AM. Pre operative treatment of 5 acromegalics with a somatostatin analogue: endrocrine and clinical observations. Acta Endrocrinol 1987;114:249-256.

16. Wilson CB. Role of surgery in the management of pituitary tumors. Neurosurg Clin North Am 1990;1:139-150. 